Small-molecule quorum quenchers to prevent Staphylococcus aureus infection

By: Nadja B. Cech, Alexander R. Horswill

Cech, N. B., Horswill, A.R. (2013). Small molecule quorum quenchers for prevention of

Staphylococcus aureus infection. Future Microbiology, 8(12), 1-4. doi:10.2217/fmb.13.134

Made available courtesy of Future Medicine: $\underline{\text { http://dx.doi.org/10.2217/fmb.13.134 }}$

***@ $\subseteq$ Future Medicine. Reprinted with permission. No further reproduction is authorized without written permission from Future Medicine.***

***Note: Full text of article below 


\section{Small-molecule quorum quenchers to prevent Staphylococcus aureus infection}
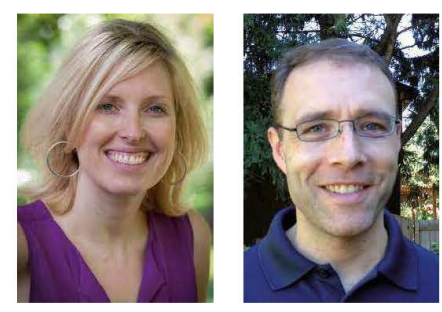

"The concept underlying antivirulence is to shut down pathogenesis mechanisms

in the invading bacteria without

impacting growth, thereby enabling the

host immune system to clear the infection in the absence of antibiotics."

Nadja B Cech' \& Alexander R Horswill ${ }^{\star 2}$

'Department of Chemistry/Biochemistry, The University of North Carolina Greensboro, 435 Sullivan Building, Greensboro, NC 27402, USA

2Department of Microbiology, Roy J and Lucille A Carver College of Medicine, University of lowa, lowa City, IA 52242, USA

*Author for correspondence: alex-horswill@uiowa.edu

Antibiotic-resistant bacteria are one of the most significant current threats to human health. The diminishing antibiotic development pipeline coupled with the propensity for bacterial pathogens to evolve resistance represents an ongoing crisis that needs to be addressed [1]. Resistance development has long been a major concern with Staphylococcus aureus, a notorious bacterial pathogen that causes a wide spectrum of acute and chronic disease. The incidence of methicillin-resistant $S$. aureus (MRSA) infections in both hospital and community settings continues to rise [2], and healthcare-associated infections due to MRSA cost more and lead to longer hospital stays than those caused by any other bacterial pathogen [3]. Antibiotic stewardship to preserve the most efficacious treatments for severe bacterial infections needs to be at the forefront of strategies designed to address growing resistance challenges. How can these principles be applied to managing $S$. aureus disease burden? When considering the breadth of infections caused by $S$. aureus, the majority are skin and soft tissue infections (SSTIs), resulting in 11.6 million ambulatory care visits per year [4]. In emergency departments, $76 \%$ of $S$. aureus cases are SSTIs and $59 \%$ of these are MRSA [5]. Many of these SSTIs would be considered uncomplicated and treated with straightforward courses of antibiotics. Considering these points, the load of antibiotic therapy being directed toward $S$. aureus SSTIs is significant, and it seems probable that this is contributing to resistance development. What if innovative countermeasures for SSTIs that do not drive resistance could be translated into approved therapies? Such a strategy could help preserve the most effective antibiotics for the severe, deep-seated $S$. aureus infections and enable uncomplicated infections to be treated more rapidly and effectively.

Antivirulence strategies represent a promising approach that could address the challenge of antibiotic stewardship [1]. The concept underlying antivirulence is to shut down pathogenesis mechanisms in the invading bacteria without impacting growth, thereby enabling the host immune system to clear the infection in the absence of antibiotics. Quorum-sensing systems in particular have drawn attention as targets for the development of virulence-controlling therapeutics [6]. Although bioactive agents that function in this manner have yet to reach the clinic, animal model studies suggest potential for the approach $[7,8]$. The strategy of disarming the pathogen could lead to treatments that do not select or enrich for bacterial resistance, while simultaneously preserving the beneficial resident flora. This article covers some of the recent advances in the discovery and development of small-molecule antivirulence agents that inhibit $S$. aureus quorum sensing.

Pathogenesis in $S$. aureus is regulated by the agr quorum-sensing system, a cell density-dependent regulator that responds to the extracellular concentration of a peptide signaling molecule (reviewed in [9]). This signaling molecule is a modified peptide (called an autoinducing peptide [AIP]), which is typically 7-9 residues in length and has its last five residues cyclized into a thiolactone ring. The AIP signal is produced from a ribosomal precursor and processed and secreted from the cell by the AgrB membrane endopeptidase and signal peptidase SpsB. Once AIP reaches a critical concentration, it binds to a

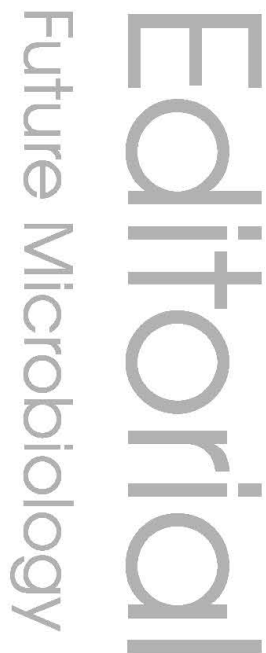

\section{Keywords}

- MRSA $=$ quorum quenching

- quorum sensing

- Staphylococcus aureus

Future Medicine ${ }_{\text {part of }}$ 
receptor on the AgrC histidine kinase, activating the kinase to phosphorylate the AgrA response regulator and inducing the quorum-sensing system. The primary output of AgrA is a large transcript called RNAIII, driven by the $\mathrm{P} 3$ promoter, and high RNAIII levels lead to the production of numerous virulence factors, including toxins, exo-enzymes and immunomodulators. Small molecules that inhibit this system (referred to as 'quorum quenchers') are the focus of this article, and three obvious targets for such compounds are AgrC, AgrA or AgrB. Of these, AgrC has been the subject of most research and development, and its extracellular location and function as the AIP receptor have drawn considerable attention. However, AgrC exhibits signs of being hypermutable [10], potentially meaning a future reservoir of resistance. By contrast, AgrA and AgrB are highly conserved among the Staphylococci and even other Gram-positive pathogens. Targeting these proteins could ultimately prove more fruitful.

"...interfering autoinducing peptlde
structures have proven effectlve for
treatment of Staphylococcus aureus skin
abscess in mouse models, suggesting the
potentlal beneflt of quorum quenching
to prevent skin and soft tlssue Infections."

The first identified quorum-quenching agents are the AIPs themselves, which are produced by S. aureus and other Staphylococcal strains, and interact directly with AgrC. Through a mechanism coined 'agr interference', the AIPs produced by one strain can cross-inhibit a strain producing a different agr class [9]. This property of the agr system has led to the identification of peptide scaffolds that act as AgrC-receptor antagonists and inhibit specific $S$. aureus strains. Such interfering AIP structures have proven effective for treatment of $S$. aureus skin abscess in mouse models [7], suggesting the potential benefit of quorum quenching to prevent SSTI. Development of AIP-based receptor antagonists continues to be an active area of exploration, and numerous inhibiting structures have been identified and optimized (recently reviewed [8]). Chemical screening has also identified synthetic agents thought to act as AgrC-receptor antagonists, such as benzbromarone, a treatment for gout. Benzbromarone is a potent quorum quencher $\left(\mathrm{IC}_{50}: 0.1-0.2 \mu \mathrm{M}\right)$ and inhibits production of agr-regulated virulence factors in all types of $S$. aureus strains [8].
The output of AgrC is the phosphorylation of the AgrA response regulator and, in turn, the upregulation of RNAIII to induce $S$, aureus virulence. Identification of agents that target ArgA directly or prevent the induction of RNAIII has yielded promising leads. The DNA-binding domain structure of the AgrA response regulator has been solved, and this structure has been used as a template for nuclear magnetic resonance-based inhibitor screening [11]. The best AgrA inhibitors were confirmed in electrophoretic mobility shift assays using purified protein. Computer docking has also yielded promising AgrA small-molecule inhibitors, which were verified in follow-up studies [12]. Using RNAIII production as an output, the synthetic smallmolecule quorum quencher named savirin, for $S$. aureus virulence inhibitor, was identified by combinatorial library screening [13]. Savirin is a potent inhibitor of RNAIII transcription $\left(\mathrm{IC}_{50}\right.$ : $\sim 1 \mu \mathrm{M}$ ) and simultaneously prevents the production of virulence factors $\alpha$-toxin and phenol soluble modulins. Most importantly, savirin attenuated MRSA in a mouse model of SSTI and showed no potential for resistance development through successive rounds of in vivo testing [13].

Another strategy of quorum quenching that has yielded preliminary success is targeting the production of the AIP signal. The Type I signal peptidase SpsB is essential for AIP biosynthesis, and linear peptide inhibitors of this peptidase were found to quench the agr system actoss strains [14]. The fungal metabolite ambuic acid was identified in screens for compounds that inhibited the production of the peptide quorum-sensing signal in Enterococcus faecalis [15]. In follow-up analysis, ambuic acid successfully prevented endopeptidase activity in the AgrB homo$\log$ FsrB, and it acted as a quorum quencher by inhibiting AIP production in $S$. aureus and other Gram positives [15].

\footnotetext{
"The concept of screening based on quorum quenching activity is relatively new to the natural products community; nonetheless, some promising findings have recently been publlshed."
}

Natural products have proven to be a rich source of anti-infective agents, with approximately $77 \%$ of drugs for this purpose derived from natural sources [16]. The concept of screening based on quorum quenching activity is relatively new to the natural products community; nonetheless, some promising findings 
have recently been published. One example is the previously mentioned AgrB inhibitor ambuic acid, which is a polyketide-derived epoxyquinone produced by fungi of Monochaetia spp. and Pestalotiopsis spp [15]. Other examples include cyclized dipeptide molecules secreted by Lactobacillus reuteri, which are thought to inhibit AgrC-A two-component action [17], and the compound norlichexanthone, which is produced by the fungus Penicillium algidum [18]. Botanical extracts and their components have also been shown to possess quorum quenching activity against $S$. aureus. In one study, multiple plant extracts were found to inhibit the production of $\delta$-toxin, a small peptide encoded in the RNAIII transcript [19], and in another, extracts from the medicinal plant goldenseal (Hydrastis canadensis) were found to inhibit RNAIII and $\alpha$-toxin production, and reduce toxicity to human skin epithelial cells [20]. In both of the aforementioned studies, however, the specific compounds responsible for activity were not identified.

Although quorum quenching in $S$. aureus has made substantial progress over the past decade, there remain many undeveloped areas that need to be addressed. AgrC receptor antagonists are the most advanced candidates, but the undesirable pharmacokinetic properties of peptide inhibitors and the penchant for $\mathrm{AgrC}$ to evolve mutations raise questions about their long-term therapeutic utility [10]. AgrB is the most unique component of the agr system across Gram-positive pathogens [9], yet ambuic acid is the only reported AgrB inhibitor, and it has only limited efficacy [15]. In all $S$. aureus strains, AgrA is arguably the most conserved component of the system and is divergent from other classes

\section{References}

1. Spellberg B, Bartlett JG, Gilbert DN. The future of antibiotics and resistance. $N$. Engl. J. Med. 368(4), 299-302 (2013).

2. Deleo FR, Chambers HF. Reemergence of antibiotic-resistant Staphylococcus aureus in the genomics era. J. Clin. Invest. 119(9), 2464-2474 (2009).

3. Zimlichman $\mathrm{E}$, Henderson $\mathrm{D}$, Tamir $\mathrm{O}$ et al. Health care-associated infections: a metaanalysis of costs and financial impact on the US health care system. JAMA Intern. Med. doi:10.1001/jamainternmed.2013.9763 (2013) (Epub ahead of print).

4. McCaig LF, McDonald LC, Mandal S, Jernigan DB. Staphylococcus aureus-associated skin and soft tissue infections in ambulatory care. Emerg. Infect. Dis. 12(11), 1715-1723 (2006).

of bacterial response regulators [11], but only a handful of moderately potent inhibitors have been identified for this target $[11,12]$. Thus, there remains significant untapped potential for both AgrB and AgrA as screening targets. Unfortunately, the mechanism of action of some of the most effective $S$. aureus quorum quenchers remains unclear, and to resolve this uncertainty and improve future screening, assays that provide more mechanistic information are needed. The limited body of currently published quorum quenching compounds includes both synthetic and natural product compounds, and there is reason to assume that both pools present possible sources of novel antivirulence therapies. Ultimately, recent successful in vivo studies suggest the promise of antivirulence strategies for addressing the immensely problematic issue of antibiotic-resistant $S$, aureus infections. The challenge of ongoing studies will be the translation of these basic research concepts into approved clinical therapies that can preserve our most important antibiotics for future use.

Financial \& competing interests dlsclosure

This work was supported by grants AT006860 (NB Cech and AR Horswill) and AT007052 (AR Horswill)

from the National Center for Complementary and Alternative Medicine, a component of the NIH. The authors have no other relevant affiliations or financial involvement with any organization or entity with a financial interest in or financial conflict with the subject matter or materials discussed in the manuscript apart from those disclosed.

No writing assistance was utilized in the production of this manuscript.

5. Moran GJ, Krishnadasan A, Gorwitz RJ et al. Methicillin-resistant $S$. aureus infections among patients in the emergency department. N. Engl. J. Med. 355(7), 666-674 (2006).

6. Gray B, Hall P, Gresham H. Targeting agrand agr-like quorum sensing systems for development of common therapeutics to treat multiple Gram-positive bacterial infections. Sensors (Basel) 13(4), 5130-5166 (2013).

7. Wright JS 3rd, Jin R, Novick RP. Transient interference with staphylococcal quorum sensing blocks abscess formation. Proc. Natl Acad. Sci. USA 102(5), 1691-1696 (2005).

8. Gordon CP, Williams P, Chan WC. Attenuating Staphylococcus auress virulence gene regulation: a medicinal chemistry perspective. J. Med. Chem. 56(4), 1389-1404 (2013).

9. Thoendel M, Kavanaugh JS, Flack CE,
Horswill AR. Peptide signaling in the staphylococci. Chem. Rev. 111(1), 117-151 (2011).

10. Somerville GA, Beres SB, Fitzgerald JR et al. In vitro serial passage of Staphylococcus aureus: changes in physiology, virulence factor production, and agr nucleotide sequence. J. Bacteriol. 184(5), 1430-1437 (2002).

11. Leonard PG, Bezar IF, Sidote DJ, Stock AM. Identification of a hydrophobic cleft in the LytTR domain of AgrA as a locus for small molecule interactions that inhibit DNA binding. Biocbemistry 51(50), 10035-10043 (2012).

12. Khodaverdian V, Pesho M, Truitt B et al. Discovery of anti-virulence agents against MRSA. Antimicrob. Agents Chemother. 57(8), 3645-3652 (2013).

13. Sully E. Small molecule inbibitor of 


\section{Editorial Cech \& Horswill}

Staphylococcus aureus virulence [PhD thesis]. University of New Mexico, NM, USA (2011).

14. Kavanaugh JS, Thoendel M, Horswill AR. A role for type I signal peptidase in Staphylococcus aureus quorum-sensing. $\mathrm{Mol}$. Microbiol. 65, 780-798 (2007).

15. Nakayama J, Uemura $Y$, Nishiguchi $K$, Yoshimura N, Igarashi Y, Sonomoto K. Ambuic acid inhibits the biosynthesis of cyclic peptide quormones in Gram-positive bacteria. Antimicrob. Agents Chemother. 53(2), 580-586 (2009).

16. Newman DJ, Cragg GM. Natural products as sources of new drugs over the 30 years from 1981 to 2010. J. Nat. Prod. 75(3), 311-335 (2012).

17. Li J, Wang W, $\mathrm{Xu} S \mathrm{~S}$, Magarvey NA, McCormick JK. Lactobacillus reuteriproduced cyclic dipeptides quench agrmediated expression of toxic shock syndrome toxin-1 in staphylococci. Proc. Natl Acad. Sci. USA 108(8), 3360-3365 (2011).

18. Nielsen A, Nielsen KF, Frees D, Larsen TO, Ingmer $\mathrm{H}$. Method for screening compounds that influence virulence gene expression in
Staphylococcus aureus. Antimicrab. Agents Chemother. 54(1), 509-512 (2010).

19. Quave CL, Plano LR, Bennett BC. Quorum sensing inhibitors of Staphylococcus aureus from Italian medicinal plants. Planta Med. 77(2), 188-195 (2011).

20. Cech NB, Junio HA, Ackermann LW, Kavanaugh JS, Horswill AR. Quorum quenching and antimicrobial activity of goldenseal (Hydrastis canadensis) against methicillin-resistant Staphylacoccus aureus (MRSA). Planta Med. 78(14), 1556-1561 (2012). 